\title{
Analysis of non-linear pulsatile blood flow in artery through a generalized multiple stenosis
}

Received: 15 June 2015 / Accepted: 25 October 2015 / Published online: 12 November 2015

(C) The Author(s) 2015. This article is published with open access at Springerlink.com

\begin{abstract}
The non-linear blood flow under the influence of periodic body acceleration through a generalized multiple stenosed artery is investigated with the help of numerical simulation. The arterial segment is simulated by a cylindrical tube filled with a viscous incompressible Newtonian fluid described by the Navier-Stokes equation. The non-linear equation is solved numerically using finite difference with the proper boundary conditions and pressure gradient that arise from the heart. The effect of Reynolds number is also discussed. Results are shown in comparison with the existing models.
\end{abstract}

Mathematics Subject Classification 65C20 - 65L12 - 76D05 - 92C10

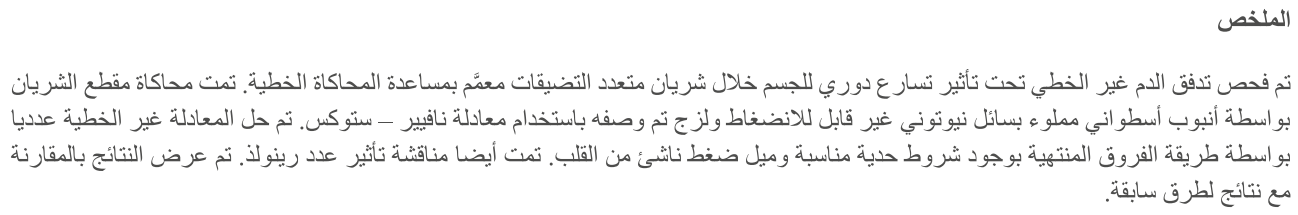

\section{Introduction}

Nowadays the investigation of blood flow analysis in a constricted stenosed artery is very important in the medical domain because of the fact that many of the diseases such as heart attacks and strokes are related to blood flow and also the physical characteristics of the vessel wall. At present the leading causes of the death in the world are due to heart diseases such as atherosclerosis. Atherosclerosis occurs where the arteries become narrowed and hardened due to an excessive build up of plaque inside the artery wall. The formation of plaque disrupts the flow of blood around the body and leads to different cardiovascular diseases. Atherosclerosis involves an accumulation of low-density lipoprotein in the wall of large arteries, typically where the wall shear rate is low and oscillatory [1]. When the cells do not get the sufficient oxygen due the reduced of flow rate in the artery, then it may produce ischemia, which is typically used as an indicator for surgical intervention such as angioplasty and bypass operations [1,2].

Modeling of blood flow through arterial multiple stenosis is very challenging. Numerous experimental and computational methods have used to quantify the velocity and wall shear stress of blood flow in human

S. Changdar $(\bowtie)$

Institute of Engineering \& Management, Saltlake, Kolkata 700101, India

E-mail: satyasaran.changdar@iemcal.com

S. De

Department of Applied Mathematics, University of Calcutta, 92, Acharya Prafulla Chandra Road,

Kolkata 700 009, India

E-mail: soumenisi@gmail.com 
arteries. Experimental methods such as magnetic resonance imaging techniques have been used to quantify blood flow for diagnosing cardiovascular disease. Accuracy of the simulation depends mainly on suitable numerical approach, realistic model geometry and boundary conditions [3]. Many investigators have focused their attention on blood flow through stenosed arteries with single stenosis. Chakravarty and Mondal [4], Lee and $\mathrm{Xu}$ [5] have pointed out that the mathematical model becomes more accurate in the presence of an overlapping stenosis instead of a mild one. Ang and Majumdar [6] have investigated asymmetric arterial blood flow with a numerical solution in three dimensions, Ikbal [7] have worked on unsteady response of non-Newtonian in magnetic field without considering periodic body acceleration effect. Kohler et al. [8] have investigated the wall shear stress with the help of magnetic resonance imaging by measuring the velocity field and comparing it with simulation outputs. Stroud et al. [9] have studied a 2D plaque model using modeling and simulation while Fischer et al. [10] worked on numerical method for the computational study of arterial blood flow with turbulence. The asymmetric flow in a symmetric sudden expansion channel have been studied using experimental and numerical techniques by Fearn et al. [11] and Durst et al. [12]. Mahapatra et al. [13] have investigated unsteady laminar separated flow through constricted channel using finite-difference technique in staggered grid distribution and suggested that the critical value of Reynolds number depends on the area reduction and the length of the constriction. Chakravarty et al. [14] have solved the blood flow model with body acceleration neglecting the non-linear terms in the model. Blood shows a non-Newtonian behaviour at low shear rate in tubes of smaller diameters. Taylor [15] suggested that at high shear rate commonly found in larger arteries blood behaves like a Newtonian fluid.

However, in this work we have worked on numerical simulations of non-linear pulsatile unsteady Newtonian blood flow in a rigid cylindrical tube through a series of multiple stenosis under the influence of periodic body acceleration. The focus of this work is to quantify blood flow in the presence of multiple stenosis. A few studies address the issue of non-linear terms which are present in Navier-Stokes equation that govern blood flow with periodic body acceleration associated with a single atherosclerotic plaque. In this paper the numerical solutions of the non-linear model are obtained by using appropriate finite difference method.

The physical assumptions of the mathematical model are covered in Sect. 2. The numerical solutions are detailed in Sect. 3. Results and discussion, including comparison of axial velocity with existing model [7] are presented in Sect. 4. Conclusions follow in Sect. 5.

\section{Physical assumptions and mathematical model}

The segment of the artery is modeled as an axisymmetric cylindrical tube with radius $r_{0}$. The blood is modeled as a homogeneous incompressible viscous unsteady Newtonian fluid of density $\rho$ and kinematic viscosity $\nu$. Therefore, the blood flow is governed by the incompressible Navier-Stokes equation.

A cylindrical coordinate system $(r, \theta, z)$ is chosen, where $(r, \theta)$ be the coordinate in the radial and circumferential direction, while $z$ is taken along the axis of artery as shown in Fig. 1. The velocity components in the axial and radial directions are $u$ and $v$ respectively. The cyclic nature of the heart pump creates pulsatile conditions in all arteries. The flow is driven by a prescribed dimensional oscillatory axial pressure gradient given by [16]

$$
-\frac{\partial p}{\partial z}=p_{0}+p_{1} \cos (\omega t), \quad t>0
$$

where $p_{0}$ and $p_{1}$ are the steady component of the pressure gradient and pulsatile component respectively. The frequency $\omega=2 \pi f, f$ is the heart pulse frequency. The pressure gradient in the radial direction is negligibly

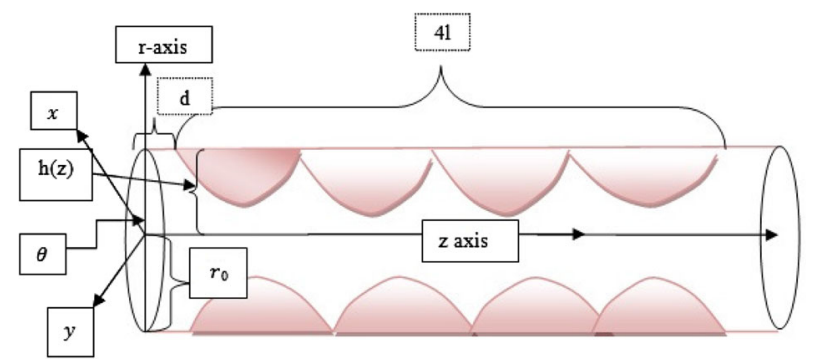

Fig. 1 Cylindrical co-ordinate system with multiple stenosis along the axial direction, where $2 l=$ the length of the stenosis and $d=$ distance of stenosis from the radial axis 
small as the lumen radius of artery is small compare to pressure wave length so that $\partial p / \partial r \simeq 0$. Also because of the human body acceleration, the axial flow is subject to an external force $F_{\text {ext }}$. For the present model, we consider the periodic force given by

$$
F_{\text {ext }}=a_{0} \cos (\omega t+\phi)
$$

where $a_{0}$ is the amplitude of the pulse.

According to the above assumptions, the blood flow dynamics is governed by the equation of continuity

$$
\frac{\partial u}{\partial z}+\frac{v}{r}+\frac{\partial v}{\partial r}=0
$$

the momentum equation in the radial direction (flow velocity $v$ )

$$
\frac{\partial v}{\partial t}=-\left(u \frac{\partial v}{\partial z}+v \frac{\partial v}{\partial r}\right)-\frac{\partial p}{\partial r}+\frac{1}{R e}\left(\frac{\partial^{2} v}{\partial r^{2}}+\frac{1}{r} \frac{\partial v}{\partial r}+\frac{\partial^{2} v}{\partial z^{2}}-\frac{v}{r^{2}}\right),
$$

and the axial direction (flow velocity $u$ )

$$
\frac{\partial u}{\partial t}=-\left(v \frac{\partial u}{\partial r}+u \frac{\partial u}{\partial z}\right)-\frac{\partial p}{\partial z}+\frac{1}{R e}\left(\frac{\partial^{2} u}{\partial r^{2}}+\frac{1}{r} \frac{\partial u}{\partial r}+\frac{\partial^{2} u}{\partial z^{2}}\right)+F_{\text {ext }} .
$$
blood.

In the above Eqs. (4) and (5), $R e=r_{0} u_{\infty} \rho / v$ is the Reynolds number, $u_{\infty}$ is the average velocity of the

Finally, to model generalized multiple axisymmetric stenosis, we define the following mathematical function $h(z)$ :

$$
h(z)=\left\{\begin{array}{cc}
1+\lambda_{1} \cos \left(\frac{\pi z}{l}\right) & d \leq z<d+l \\
1-\lambda_{2} \cos \left(\frac{\pi z}{l}\right) & d+l \leq z<d+2 l \\
1+\lambda_{3} \cos \left(\frac{\pi z}{l}\right) & d+2 l \leq z<d+3 l \\
\vdots & \\
1+(-1)^{n+1} \lambda_{n} \cos \left(\frac{\pi z}{l}\right) & d+n l \leq z \leq d+(n+1) l \\
1 & \text { otherwise }
\end{array}\right.
$$

where $\lambda_{i}, i=1 \ldots n$ are dimensionless constants representing the amplitude of the stenosis and the geometry of this axisymmetric stenosis in the cross section of the artery is shown in Fig. 2.

We numerically simulate Eqs. (3)-(5) subject to the following initial condition

$$
u(r, z, t)=0 \text { and } v(r, z, t)=0 \text { at } t=0,
$$

and the no slip boundary conditions

$$
\begin{gathered}
\frac{\partial u(r, z, t)}{\partial r}=0 \text { and } v(r, z, t)=0 \text { at } r=0 \\
u(r, z, t)=0=v(r, z, t) \text { at } r=h(z) .
\end{gathered}
$$

\section{Numerical simulation: computational method}

The finite difference scheme is used to study the non linear dynamics of blood flow through the cylindrical shape artery. To employ this method, first we transform our cylindrical domain into the rectangular domain by using the following radial transformation [17]

$$
x=\frac{r}{h(z)} .
$$

Under this transformation, the equation of continuity (3), the equations of motion in the radial direction (4) and axial direction (5), respectively, re-written as

$$
\frac{\partial u}{\partial z}+\frac{v}{x h(z)}+\frac{1}{h(z)} \frac{\partial v}{\partial x}-\frac{x}{h(z)} \frac{\partial u}{\partial x} \frac{\mathrm{d} h}{\mathrm{~d} z}=0,
$$



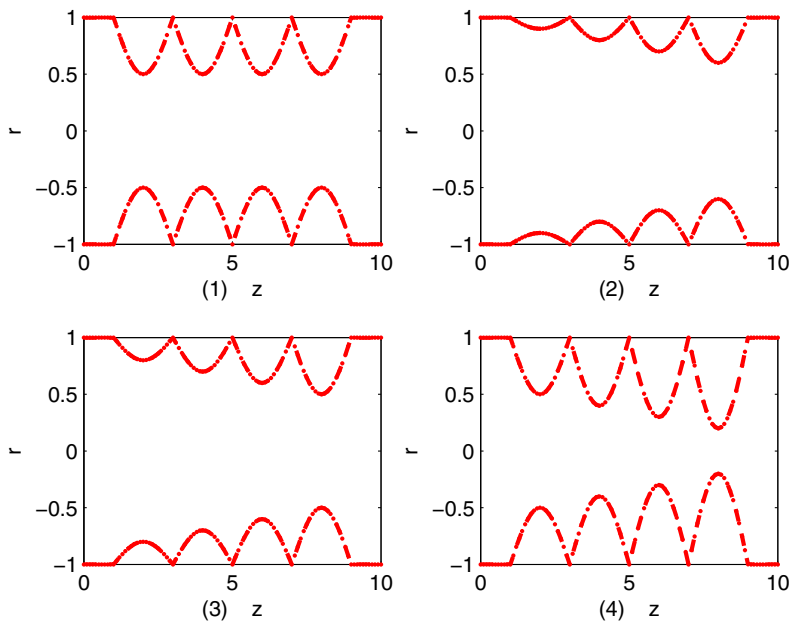

Fig. 2 The schematic representation of the axisymmetric stenosis along the axial direction as given by $h(z)$. The geometrical parameters are: (1) $n=4, l=2.00, d=1.00$ and $\lambda_{1}=0.500, \lambda_{2}=0.500, \lambda_{3}=0.500, \lambda_{4}=0.500 \quad$ (2) $n=4, l=2.00, d=1.00$ and $\lambda_{1}=0.100, \lambda_{2}=0.200, \lambda_{3}=0.300, \lambda_{4}=0.400$ (3) $n=4, l=2.00, d=5.00$ and $\lambda_{1}=0.200, \lambda_{2}=0.300, \lambda_{3}=0.400, \lambda_{4}=0.500$ (4) $n=4, l=2.00, d=1.00$ and $\lambda_{1}=0.500, \lambda_{2}=0.600, \lambda_{3}=$ $0.700, \lambda_{4}=0.800$

$$
\begin{aligned}
\frac{\partial v}{\partial t}= & -\left(\frac{v}{h(z)} \frac{\partial v}{\partial x}+u \frac{\partial v}{\partial z}-\frac{x u}{h(z)} \frac{\partial v}{\partial x} \frac{\mathrm{d} h}{\mathrm{~d} z}\right)+\frac{1}{R e}\left\{\frac{1}{h^{2}(z)}\left(\frac{\partial^{2} v}{\partial x^{2}}+\frac{1}{x} \frac{\partial v}{\partial x}-\frac{v}{x^{2}}\right)+\frac{\partial^{2} v}{\partial z^{2}}\right\} \\
& -\frac{1}{R e}\left\{\frac{2 x}{h(z)} \frac{\mathrm{d} h}{\mathrm{~d} z} \frac{\partial^{2} v}{\partial x \partial z}+\frac{x}{h(z)} \frac{\partial v}{\partial x} \frac{\mathrm{d}^{2} h}{\mathrm{~d} z^{2}}-\left(\frac{\frac{\mathrm{d} h}{\mathrm{~d} z}}{h(z)}\right)^{2}\left(2 x \frac{\partial v}{\partial x}+x^{2} \frac{\partial^{2} v}{\partial x^{2}}\right)\right\}
\end{aligned}
$$

and

$$
\begin{aligned}
\frac{\partial u}{\partial t}= & -\left(\frac{v}{h(z)} \frac{\partial u}{\partial x}+u \frac{\partial u}{\partial z}-\frac{x u}{h(z)} \frac{\partial u}{\partial x} \frac{\mathrm{d} h}{\mathrm{~d} z}\right)-\frac{\partial p}{\partial z}+\frac{1}{\operatorname{Re}}\left\{\frac{1}{h^{2}(z)}\left(\frac{\partial^{2} u}{\partial x^{2}}+\frac{1}{x} \frac{\partial u}{\partial x}\right)+\frac{\partial^{2} u}{\partial z^{2}}\right\} \\
& -\frac{1}{\operatorname{Re}}\left\{\frac{2 x}{h(z)} \frac{\mathrm{d} h}{\mathrm{~d} z} \frac{\partial^{2} u}{\partial x \partial z}+\frac{x}{h(z)} \frac{\partial u}{\partial x} \frac{d^{2} h}{d z^{2}}-\left(\frac{\frac{\mathrm{d} h}{\mathrm{~d} z}}{h(z)}\right)^{2}\left(2 x \frac{\partial u}{\partial x}+x^{2} \frac{\partial^{2} u}{\partial x^{2}}\right)\right\}+F_{\text {ext }}
\end{aligned}
$$

The initial condition (6) and no-slip boundary condition (7) due to the radial transformation (8) then become

$$
u(x, z, t)=0 \text { and } v(x, z, t)=0 \text { at } t=0,
$$

and

$$
\begin{array}{r}
\frac{\partial u(x, z, t)}{\partial x}=0 \text { and } v(x, z, t)=0 \text { at } x=0 \\
u(x, z, t)=0=v(x, z, t) \text { at } x=1 .
\end{array}
$$

Here, we describe the methodology used to obtain numerical solutions of (9)-(11). We use a simple grid in order to discretize these equations using the finite-difference method. The solution methodology employs the following procedure. First of all we have applied the finite difference discretization scheme to solve the nonlinear model Eqs. (9)-(11). We use the central difference approximation to discretize the spatial derivatives and the explicit forward finite difference approximation to discretize the time derivative in the following manner:

$$
\begin{aligned}
& \frac{\partial u}{\partial z}=\frac{(u)_{i+1, j}^{n}-(u)_{i-1, j}^{n}}{2 \Delta z}, \frac{\partial^{2} u}{\partial z^{2}}=\frac{(u)_{i+1, j}^{n}-2(u)_{i, j}^{n}+(u)_{i-1, j}^{n}}{\Delta z^{2}} \\
& \frac{\partial u}{\partial x}=\frac{(u)_{i+1, j}^{n}-(u)_{i-1, j}^{n}}{2 \Delta x}, \frac{\partial u}{\partial t}=\frac{(u)_{i, j}^{n+1}-(u)_{i, j}^{n}}{\Delta t}
\end{aligned}
$$


where

$$
\begin{aligned}
(u)_{i, j}^{n} & =u\left(x_{j}, z_{i}, t_{n}\right) \text { and } \\
z_{i} & =(i-1) \Delta z, i=1,2, \ldots M+1 \\
x_{j} & =(j-1) \Delta x, j=1,2, \ldots N+1 \\
t_{n} & =(n-1) \Delta t, n=1,2, \ldots
\end{aligned}
$$

Similarly we approximate all the partial derivatives of $v$. The axial velocity $(u)_{i, j}^{n}$ is obtained from Eqs. (9) and (11) by applying the above finite difference scheme at any point $\left(z_{i}, x_{j}\right)$ in the domain of interest at any time $t_{n}$ with the help of the following discretize initial and boundary conditions (discretization of the Eqs. (12) and (13))

$$
\begin{gathered}
(u)_{i, j}^{1}=0,(v)_{i, j}^{1}=0 \\
(u)_{i, 1}^{n}=(u)_{i, 2}^{n},(v)_{i, 1}^{n}=0,(u)_{i, N+1}^{n}=(v)_{i, N+1}^{n}=0,
\end{gathered}
$$

subject to the input pressure gradient and external force $F_{\text {ext }}$ from relations (1) and (2). The radial velocity $(v)_{i, j}^{n}$ is obtained from (9) and (10). The vessel walls were assumed to be rigid for all simulations within this study.

Finally, we determine the volumetric flow rate

$$
Q=2 \pi \int_{0}^{h} r u \mathrm{~d} r=2 \pi h(z)^{2} \int_{0}^{1} x u \mathrm{~d} x
$$

and the wall shearing stress [1]

$$
\tau=-\left.\mu \frac{\mathrm{d} u}{\mathrm{~d} r}\right|_{r=h(z)}=-\frac{\mu}{h(z)} \frac{\mathrm{d} u}{\mathrm{~d} x}
$$

in the rectangular domain with the help of the transformation (8), where $\mu$ is the viscosity. The discretize version of $Q$ and $\tau$ are given by the following equations

$$
(Q)_{i}^{n}=2 \pi\left(h_{i}^{n}\right)^{2} \int_{0}^{1} x_{j}(u)_{i, j}^{n} \mathrm{~d} x_{j}
$$

and

$$
\tau_{i}^{n}=-\frac{\mu}{h_{i}^{n}}\left(\frac{u_{i, N+1}-u_{i, N}}{\Delta x}\right)
$$

\section{Simulation results and discussions}

In this section we have analyzed the numerical simulation of the non-linear equations to study the influence of series of stenosis and body acceleration on the blood flow for different values of the physical parameters. The vessel walls are assumed to be rigid for all simulations within this study, with the no-slip boundary condition applied at the walls. The simulation parameters are [7,14]: $n=4.00, l=2.00, d=1.00$, $\lambda_{1}=0.50, \quad \lambda_{2}=0.50, \quad \lambda_{3}=0.50, \quad \lambda_{4}=0.50, \quad R e=400,800, a_{0}=1, \quad \omega=0.02, \quad p_{0}=0.10$, $p_{1}=0.20 \times p_{0}$.

The various grid sizes are taken in explicit finite difference scheme in order to achieve the convergence and stability of the iterative model. We have performed the experiments for grid size $65 \times 65$ and $100 \times 100$ with $\mathrm{d} t=0.01$ and 0.001 . The results are found to be very similar in both the cases.

The velocity profiles are of interest since they provide a detailed description of the flow field. The behaviors of the axial velocity profile of the blood at time $t=10$ with and without body acceleration are presented in Fig. 3a, b, respectively. Both the figures are drawn at $R e=400$ and $R e=800$ for quantifying axial velocity profile. The comparative study between the figures (Fig. 3a, b) reveals that the body acceleration enhances the axial velocity. The curves in these two figures reveal that the velocity profile is constant for $0 \leq x \leq x_{m}$ and then velocity decreases and finally goes to zero on the constricted arterial wall. The values of $x_{m}$ depends on 


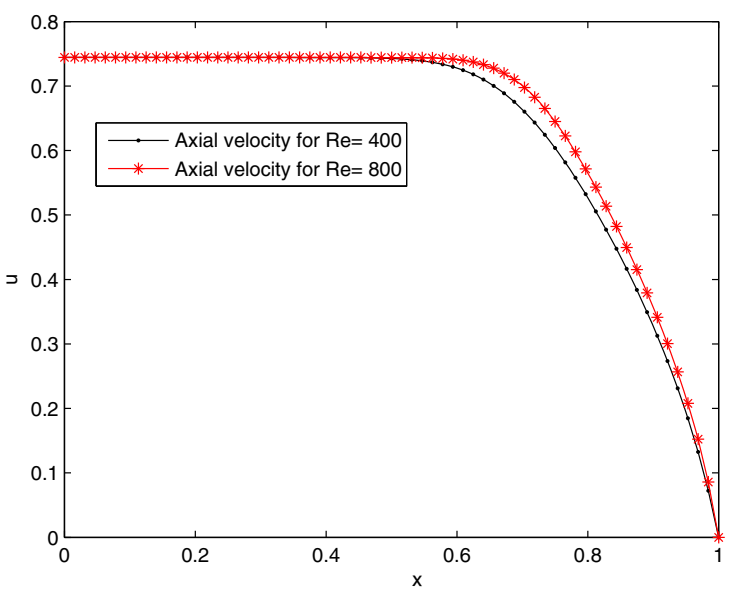

(a)

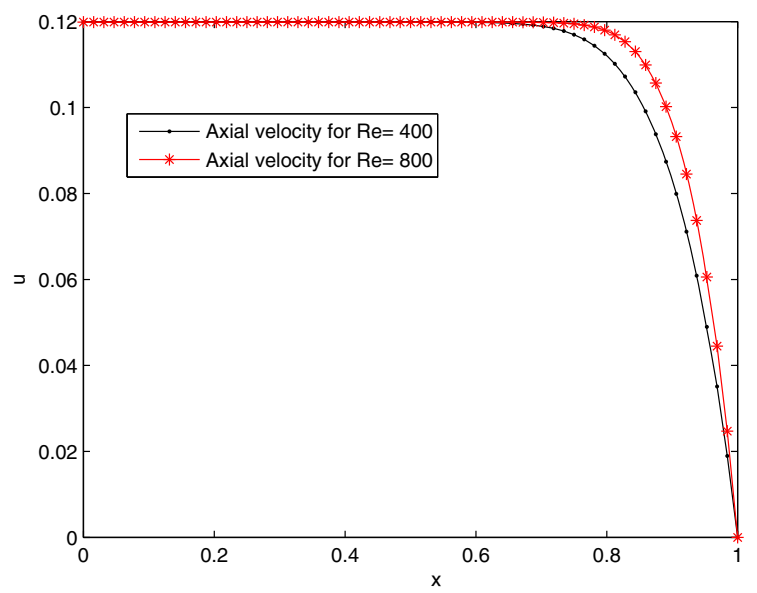

(b)

Fig. 3 a Distribution of axial velocities with body acceleration for different Reynolds number. b Distribution of axial velocities without body acceleration for different Reynolds number

both body acceleration and stenosed zone. Also in the stenosed zone of the artery ( $x \geq 0.600)$, the velocity is low. In presence of body acceleration, the velocity sharply decreases in the stenosed zone. Figure $3 \mathrm{a}, \mathrm{b}$ exhibit that at same time instance the velocity profile in case of with body acceleration is much lower than in case of without body acceleration. Thus analyzing the characteristics of the curves of the current figure, one may conclude that the applied body acceleration affects the axial velocity of the streaming blood past multiple constrictions in artery significantly and hence these applied external forces deserve to have their own importance in the present model under consideration.

Figure 4a shows the results for the distribution of axial velocity over the stenosed artery for different radial positions at Reynolds number 400. It is very clear from this figure that as blood enters into the stenosed zone, the axial velocity starts decreasing from its maximum value in non stenosed zone, until the first constriction attains its maximum value near $z=3$; it gradually increases till $z=4$ again it starts decreasing till the maximum height of the second stenosis is reached and gradually start increasing thereafter and finally flows with maximum velocity in non stenosis region. The three curves here exhibit that the axial velocity decreases in the constricted part of the artery as we move towards the arterial wall under the influence of the body acceleration. Figure $4 \mathrm{~b}$ represents the result for the distribution of the axial velocity in multiple stenosed artery for different radial positions for $R e=400$ without any external force. Examining the behaviour of the result of the present figures, one can easily quantify the effect of body acceleration passing through multiple stenosed artery.

The curves in Fig. 5a, b describes the nature of radial velocity for two different Reynolds numbers. It is observed that the radial velocity at $t=10$ in present figure is negative. It shows that at the downstream, the direction of the flow becomes opposite near the arterial wall and it causes separation in the flow field. If we think about the physiological point of view, this observation is quite justified, where the body acceleration plays a main role in order to characterise the flow under investigation.

Figure $6 \mathrm{a}, \mathrm{b}$ exhibits the variation of velocity in the radial direction for $R e=400$ at different radial positions with or without body acceleration respectively.

To test the effects body acceleration on axial and radial velocity profile, several simulations have been carried out using the contour plot as shown in Fig. 7a-d, respectively. The velocity profile $u$ and $v$ are shown in different regions with different colors representing the value of velocity with the help of color bars. One can conclude from these plots that velocity profile is segregated into different layers due to the constriction of the artery and changes, similarly it can also be observed in case of without body acceleration.

Wall shear stress plays an important role in the formation of arteriosclerosis. So it is important to study the wall shear stress distribution in the multiple stenosed artery. Figure 8a, b shows the distribution of wall shear stress on the arterial segment for two different Reynolds numbers. The wall shear stress increases rapidly near to the peak of the constriction. Here the effects of Reynolds numbers can be observed from the present figure. The wall shear stress increases as Reynolds number increases. Figure 9a, b shows the distribution of flux over the stenosed artery for different Reynolds numbers. One can conclude that flux decreases near the peak of 


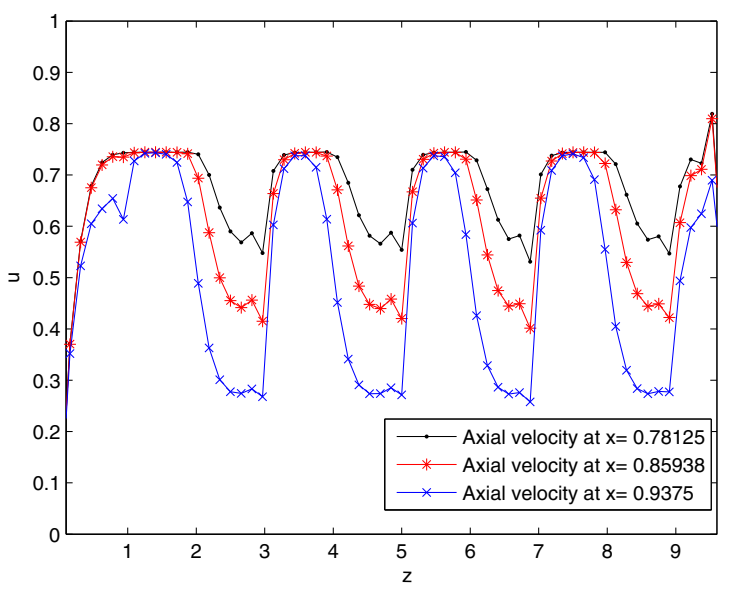

(a)

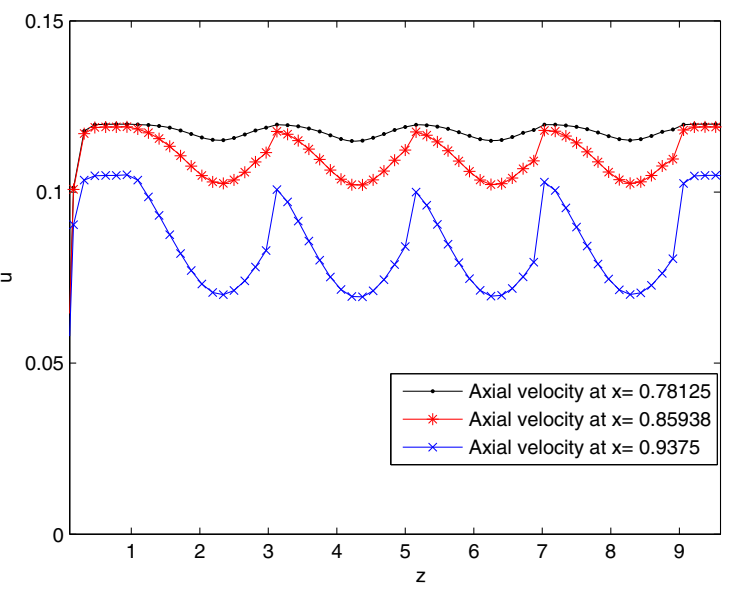

(b)

Fig. 4 Distribution of $\mathbf{a}$ axial velocities with body acceleration $\mathbf{b}$ axial velocities without body acceleration for different radial positions at time $t=10.0$

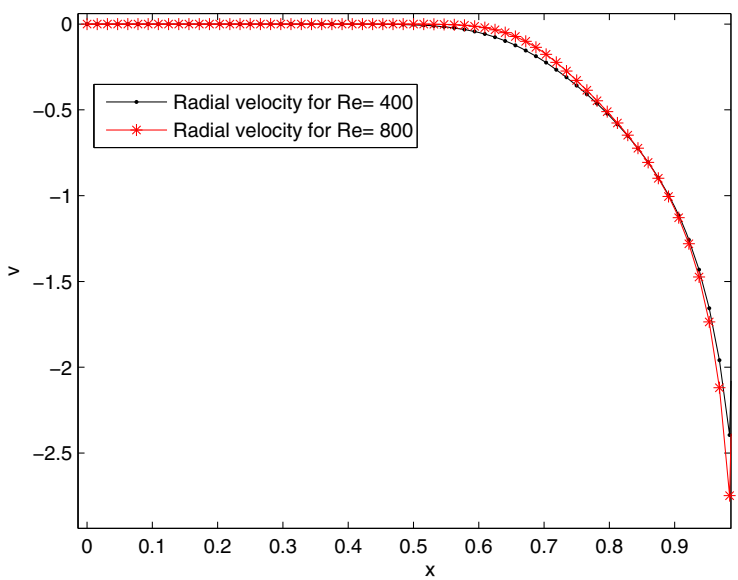

(a)

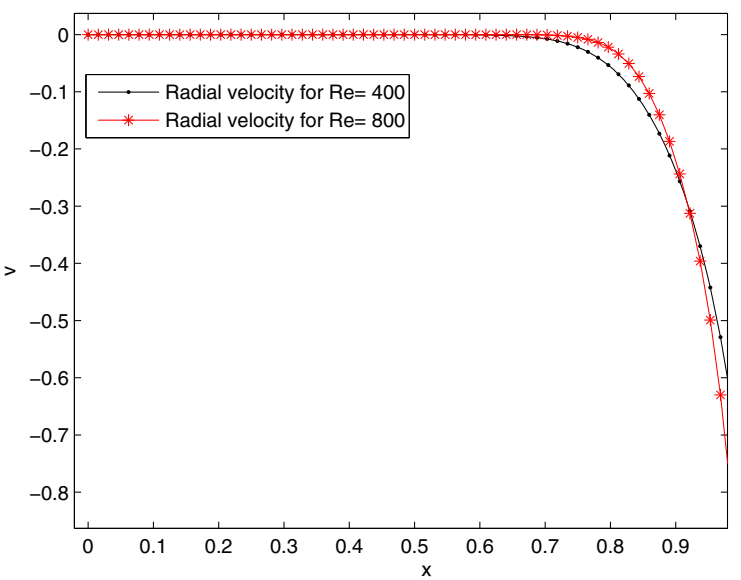

(b)

Fig. 5 Distribution of a radial velocities for varies Reynolds number with body acceleration, $\mathbf{b}$ radial velocities for different Reynolds number without body acceleration

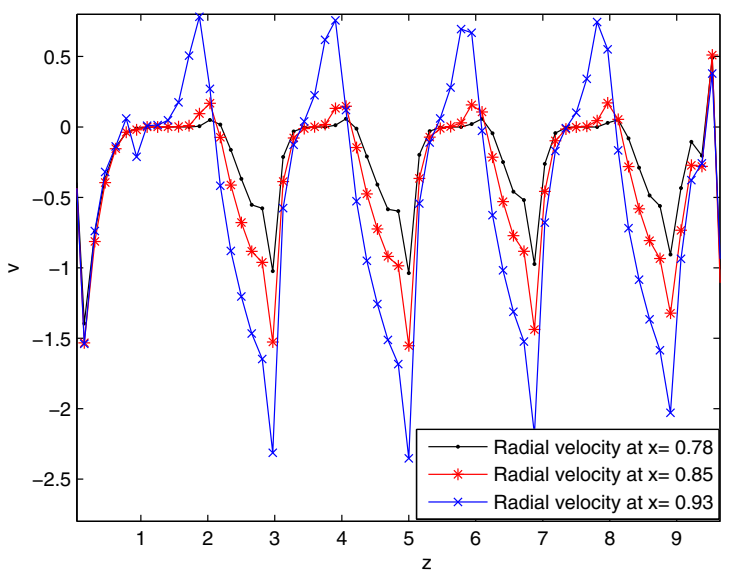

(a)

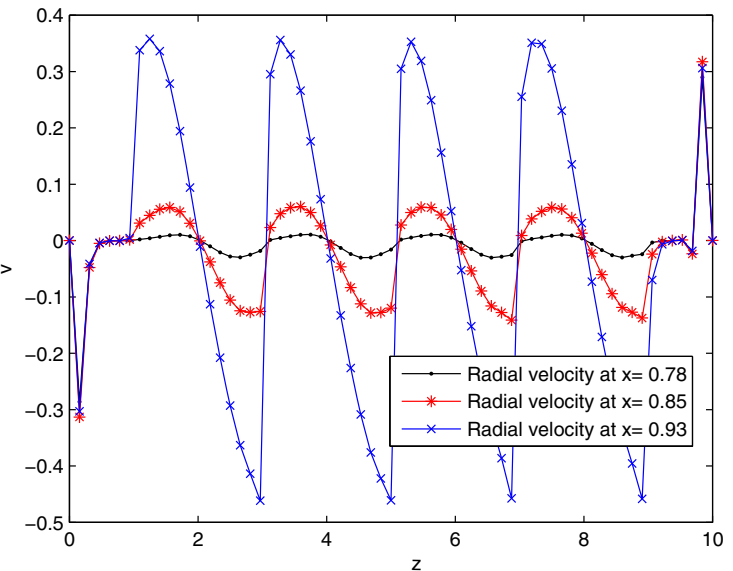

(b)

Fig. 6 Distribution of a radial velocities for varies $x$ with body acceleration, $\mathbf{b}$ radial velocities without body acceleration 


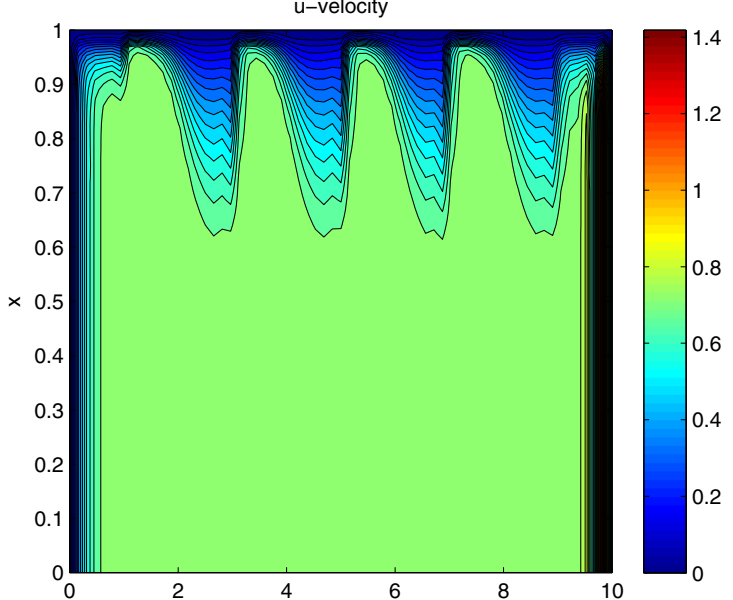

(a)

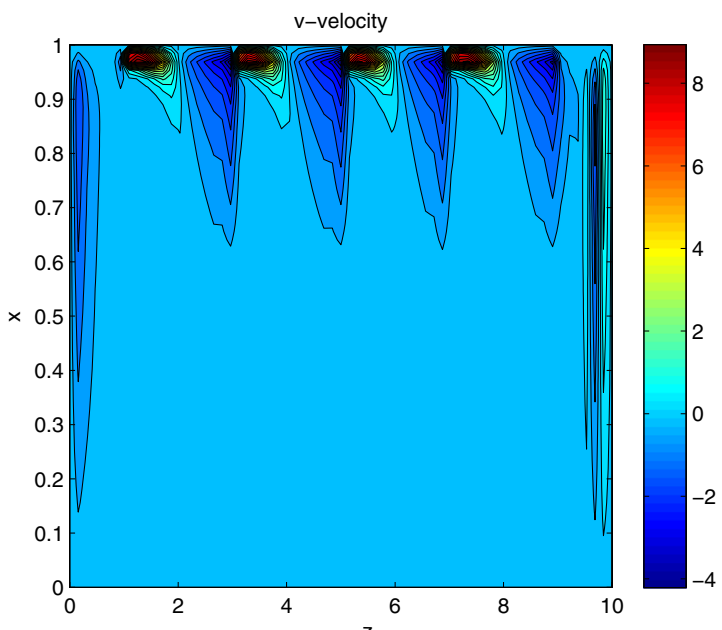

(c) ${ }^{2}$

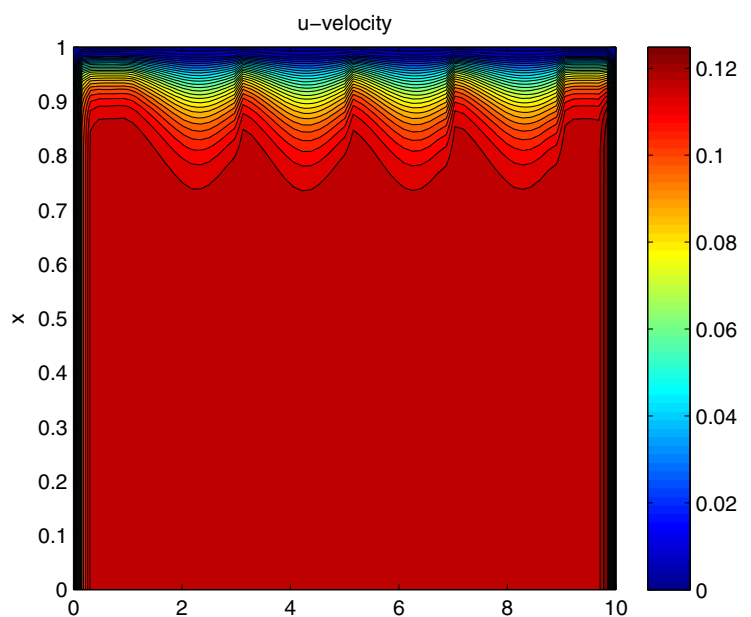

(b)

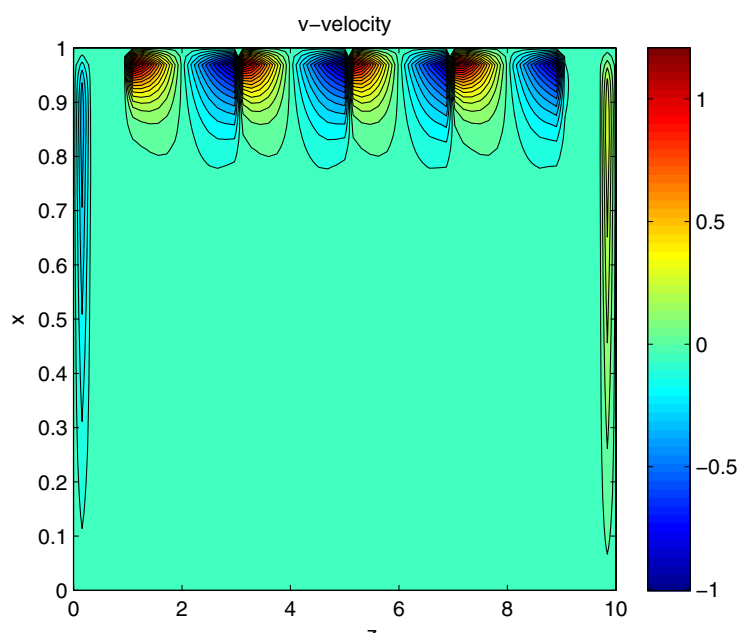

(d)

Fig. 7 Contour distribution of a axial velocities for Reynolds number $R e=400$ with body acceleration, $\mathbf{b}$ axial velocities for $R e=400$ without body acceleration, $\mathbf{c}$ radial velocities for Reynolds number $R e=400$ with body acceleration, $\mathbf{d}$ radial velocities for $R e=400$ without body acceleration

the stenosis. The streamlines of the blood flow in the artery with multi-stenosis is found in the transformed rectangular domain with grid $65 \times 65$ in the upper half zone in $z-x$ plane. We have plotted the streamlines in Fig. 10a, b for $R e=400$, where $n=2$ and $n=4$. The streamlines are observed in the linear path near the axis, which gradually gets disturbed more towards the wall of the stenosed artery. It is seen that many flow lines are attracted towards the constricted arterial wall upstream with the formation of circulation zones while others pass through the constricted region directly following the main flow. It also shows the influence of more number of stenosis for $n=4$ on the flow. Here the blood flow is more disturbed than for $n=2$. The effect of multiple stenosis is observed from the current figure. The streamline path get more disturbed near the four peaks.

Figure 11 shows the streamline patterns for $R e=400$ when the artery is free from stenosis. It is observed that the lines are parallel to the axial direction.

For the purpose of model validation, the axial velocity profile is compared with [7], as shown in Fig. 12, the results are found to be in good agreement, though their studies were based on the stenotic blood flow in which the streaming blood was treated as non-Newtonian fluid in magnetic field. The result agrees also qualitatively well for Newtonian fluid Tu et al. with [18]. 


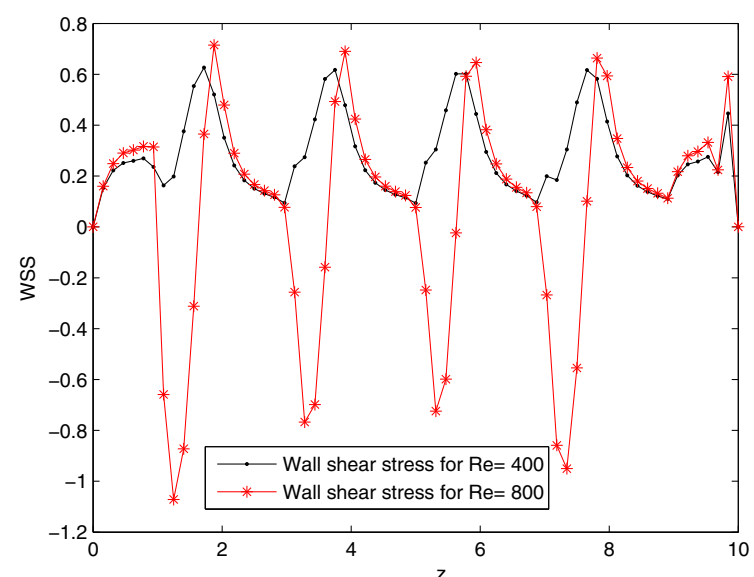

(a)

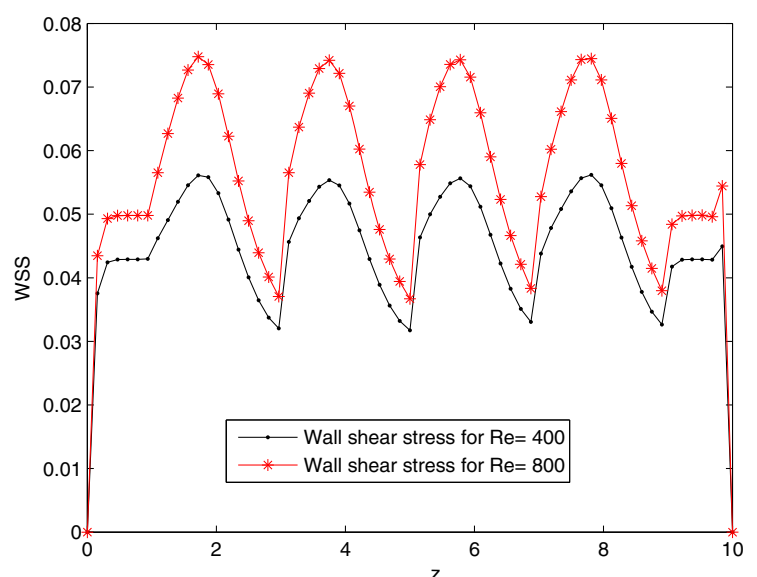

(b)

Fig. 8 Distribution of a wall shear stress with body acceleration, $\mathbf{b}$ wall shear stress without body acceleration

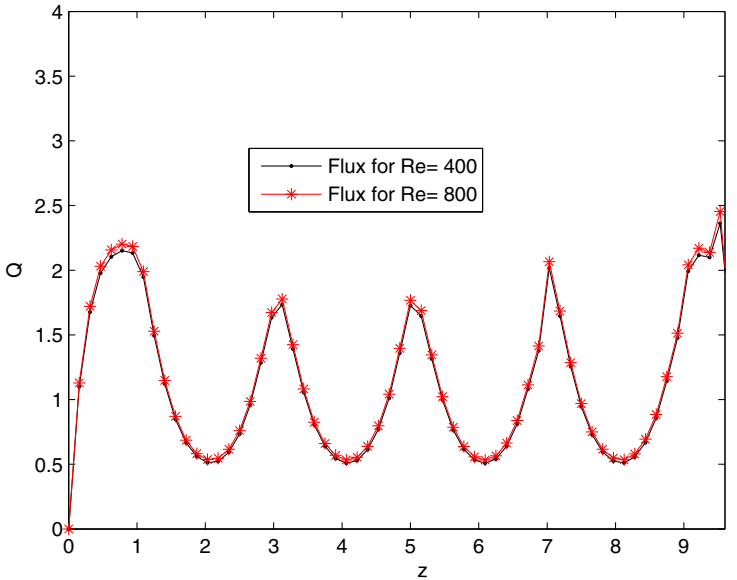

(a)

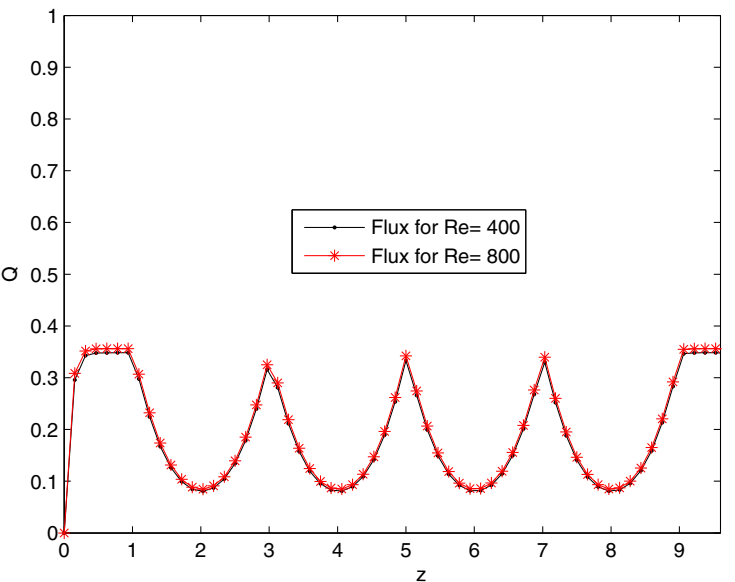

(b)

Fig. 9 Distribution of a flux with body acceleration. b Flux without body acceleration
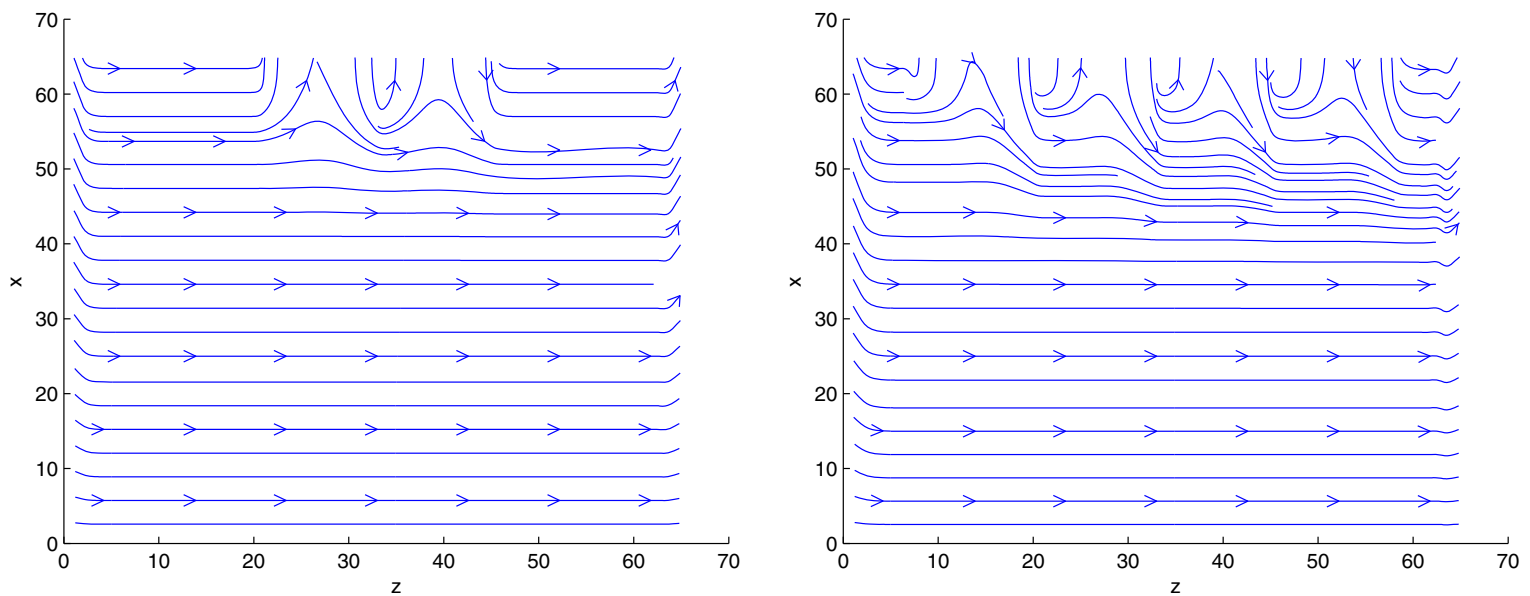

Fig. 10 Distribution of a streamlines for $n=2$. b Streamlines for $n=4$ in the upper half segment of the artery 


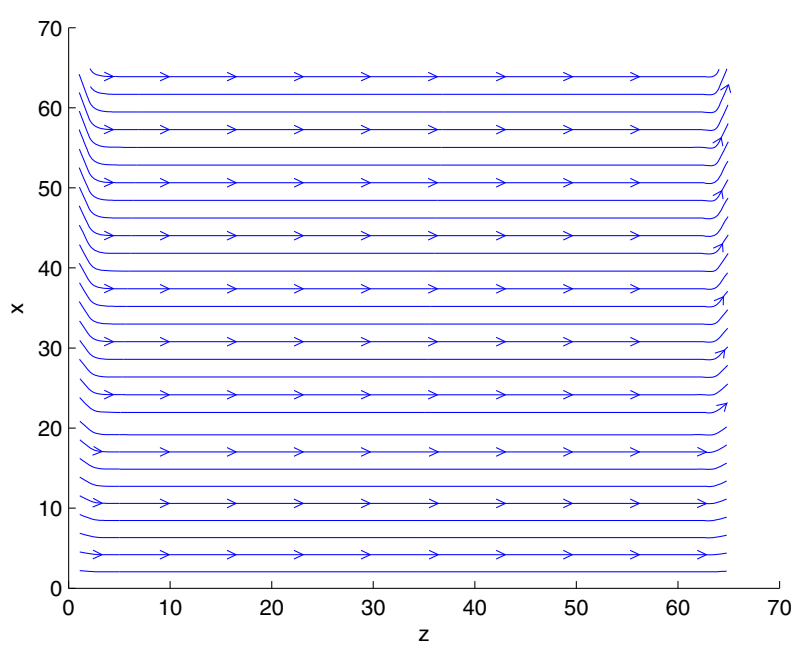

Fig. 11 The streamline patterns of the flow for $R e=400$ when there is no constriction

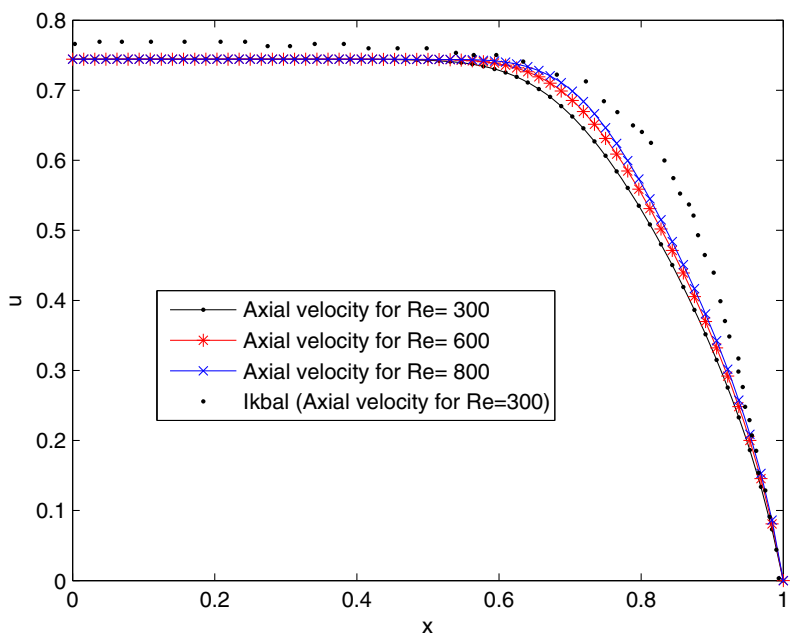

Fig. 12 Validation of axial velocity for $R e=300$

\section{Conclusions}

A non-linear mathematical model for blood flow in a multiple stenosed arterial segment has been developed under the influence of the body acceleration passing through a generalised multiple stenosis. The numerical simulation of blood flow is investigated in this study. The flow mechanism has been made, governed by a pulsatile pressure gradient. As the Reynolds number increases, the wall shear stress also increases. The flow velocity diminishes downstream from its value at the onset of the stenosis and further increases upstream towards the non-stenosed region. Volumetric flow rate occurs significantly where the arterial narrowing approaches maximum. The streamline patterns show the distinct boundary layer characteristics in the arterial segment. This is validated by the flow visualisation observed by the simulation studies of [7]. The results obtained here would help the people such as pathologists and researchers greatly in gaining better insight into blood flow models through the generalised multiple stenosis artery under the influence of body acceleration.

Acknowledgments The authors thank the reviewers for important comments and suggestions to revise and improve the manuscript. The authors are grateful to Dr. Samiran Ghosh, Department of Applied Mathematics, University of Calcutta, for fruitful discussions. This work is partially supported by CPEPA, UGC, New Delhi.

Open Access This article is distributed under the terms of the Creative Commons Attribution 4.0 International License (http:// creativecommons.org/licenses/by/4.0/), which permits unrestricted use, distribution, and reproduction in any medium, provided 
you give appropriate credit to the original author(s) and the source, provide a link to the Creative Commons license, and indicate if changes were made.

\section{References}

1. Wooton, D.M.; Ku, D.N.: Fluid mechanics of vascular systems, diseases and thrombosis. Annu. Rev. Biomed. Eng. 1, 299-329 (1999)

2. Taylor, C.A.; Draney Mary, T.: Experimental and computational methods in cardiovascular fluid mechanics. Annu. Rev. Fluid Mech. 36, 197-231 (2004)

3. Fang, B.; Zhu, L.; Fok, P.; Lu, X.: Simulation of a pulsatile non-Newtonian flow past a stenosed 2D artery with atherosclerosis. Comput. Biol. Med. 43, 1098-1113 (2013)

4. Chakravarty, S.; Mondal, P.K.: Mathematical modelling of blood flow through an overlapping arterial stenosis. Math. Comput. Model. 19, 59-70 (1994)

5. Lee, K.W.; Xu, X.Y.: Modelling of Flowand wall behaviour in a mildly stenosed tube. Med. Eng. Phys. 24, 575-586 (2002)

6. Ang, K.C.; Mazumdar, J.N.: Mathematical modelling of three-dimensional flow through an asymmetric arterial stenosis. Math. Comput. Model. 25, 19-29 (1997)

7. Ikbal, Md.A.; Chakravarty, S.; Wong, K.K.L.; Majumdar, J.; Mandal, P.K.: Unsteady response of non-Newtonian blood flow through a stenosed artery in magnetic field. J. Comput. Appl. Math. 230, 243-259 (2009)

8. Kohler, U.; Marshall, I.; Robertson, M.B.; Long, Q.; Xu, X.Y.: MRI measurement of wall shear stress vectors in bifurcation models and comparis on with CFD predictions. J. Magn. Reson. Imaging. 14, 563-573 (2001)

9. Stroud, J.S.; Berger, S.A.; Saloner, D.: Numerical analysis of flow through a severely stenotic carotid artery bifurcation. J. Biomech. Eng. 124, 9-20 (2002)

10. Fischer, P.F.; Loth, F.; Lee, S.E.; Lee, S.W.; Smith, D.; Bassiouny, H.: Simulation of high Reynolds number vascular flows. Comput. Meth. Appl. Mech. Eng. 196, 3049-3060 (2007)

11. Fearn, R.M.; Mullin, T.; Clile, K.A.: Nonlinear flow phenomena in a symmetric sudden expansion. J. Fluid. Mech. 211, 595-608 (1990)

12. Durst, F.; Pereira, J.C.; Tropea, C.: The plane symmetric sudden expansion. J. Fluid Mech. 248, 567-581 (1993)

13. Mahapatra, T.; Layek, G.C.; Maiti, M.K.: Unsteady laminar separated flow through constricted channel. Int. J. Non-Linear Mech. 37, 171-186 (2002)

14. Chakravarty, S.; Sannigrahi, A.K.: Effect of body acceleration on blood flow in an irregular stenosed artery. Math. Comput. Model. 19,93-103 (1994)

15. Taylor, M.G.: The influence of anomalous viscosity of blood upon its oscillatory flow. Phys. Med. Biol 3, 273-290 (1959)

16. Burton, A.C.: Physiology and Biophysics of the Circulation, Introductory Text. Year Book Medical Publisher, Chicago (1966)

17. Ling, S.C.; Atabek, H.B.: A nonlinear analysis of pulsatile flow in arteries. J. Fluid. Mech. 55, 493-511 (1972)

18. Tu, C., Deville, M., Dheur, L., Vanderschuren, L.: Finite element simulation of pulsatile flow through arterial stenosis. J. Biomech. 25, 1141-1152 (1992) 